\title{
Adoptive Lamps Based on the Pattern Recognition
}

\author{
Ya Mou \\ North China Electric Power University, Beijing, 102206 \\ hunter2011@foxmail.com
}

Keywords: Pattern Recognition, Cascade Adaboost Algorithm Open CV Human Eye detection

\begin{abstract}
Since the pattern recognition technology has been put forward in 1960s, it has attracted many researchers' in-depth exploration and research because of its great development potential. Over the years, the pattern recognition has made great progress in the field of face recognition, fatigue detection, human-computer interaction and so on. The purpose of this paper is to design a system that can automatically detect the human eye state and make the corresponding feedback. The system can reduce the fatigue and protect the eyes. When there is a slight change in the light, the eye will make the corresponding change. When the eye is in a comfortable state, the light is at a suitable value.
\end{abstract}

\section{Introduction}

The software used in this paper is the combination of VS2013 and OpenCV. The system is built by calling OpenCV library function. In this paper, a cascade Adaboost algorithm is used for face detection, eye detection and pupil localization. Face detection in the input image, human eye detection and pupil location based on face detection, in order to find the diameter of the pupil and iris diameter. Through the serial port, the ratio of the diameter of the iris and pupil diameter is transferred in accordance with certain mapping relation to the analog-to-digital conversion module, and output different voltage controls the brightness of LED. Then supplemented with feedback adjustment mechanism, to ensure that the light emitted by the lamp is the light that the human eye demands.

\section{Background}

In the information big data era, people often use the electronic products in inappropriate light, resulting in a straight rise of incidence of eye diseases. To solve the problem is to adjust the light to the eye. And there is no recognized standards for the light in the world at present, in this case, the human definition of light brightness and light passive adjustment cannot improve the current electronic products over use situation, where eye disease incidence is increasing year by year. We are based on the concept of healthy living, applied the pattern recognition technology to the lamp, and are devoted to the study of adaptive lighting Table lamp can be adjusted according to the changes of the environment and the state of the human eye to make the human eye feel comfortable state, thereby reducing the damage to the eyes and improve the health of the eye.

\section{Overall Design}

In the process of realizing the adaptive adjustment of light, the Adaboost algorithm and cascade classifier in the field of pattern recognition are mainly used.

Pattern Recognition. Pattern recognition is a class attribute sample (model) to determine the process, which is a sample belonging to a type in a variety of types, and since 1920s, it has been applied to the biometric authentication, fingerprint recognition, voice recognition, speech recognition, digital watermarking etc. Pattern recognition technology is the basis of artificial intelligence. We use the camera to shoot pictures, through the Adaboost algorithm and cascade classifier to identify the state of the eye, and determine the eye on the demand for light.

Haar Integral Image. Backlit photos shot by the infrared camera converted to grayscale. In or- 
der to obtain the characteristic of grayscale values, the input image is scanned by point. I (x, y) is the gray value of pixels $(\mathrm{x}, \mathrm{y})$ in the input image. If an image is assumed, at a certain point $(\mathrm{x}, \mathrm{y})$, the gray value of the pixel is calculated, and the longitudinal coordinates are calculated to be less than or equal to the $\mathrm{Y}$ of all pixels. The gray value of the pixel is defined as $\mathrm{s}(\mathrm{x}, \mathrm{y})$, and the integral map of the image is obtained by using the recursion formula:

$$
\begin{gathered}
s(\mathrm{x}, \mathrm{y})=\sum_{y^{\prime} \leq y} I\left(\mathrm{x}, \mathrm{y}^{\prime}\right) \\
s(\mathrm{x}, \mathrm{y})=s(\mathrm{x}, \mathrm{y}-1)+\mathrm{i}(\mathrm{x}, \mathrm{y}) \\
i i(\mathrm{x}, \mathrm{y})=\mathrm{ii}(\mathrm{x}-1, \mathrm{y})+\mathrm{s}(\mathrm{x}, \mathrm{y})
\end{gathered}
$$

which $x$ and $y$ starts from $0, s(x, y-1)=0, i i(x-1, y)=0$. The gray integral of all the pixels in the rectangle region can be obtained by the integral graph. The Integral image ii of Point 1 is: $\mathrm{ii}=\operatorname{sum}(\mathrm{A})$, specific integral range is showing in the figure.

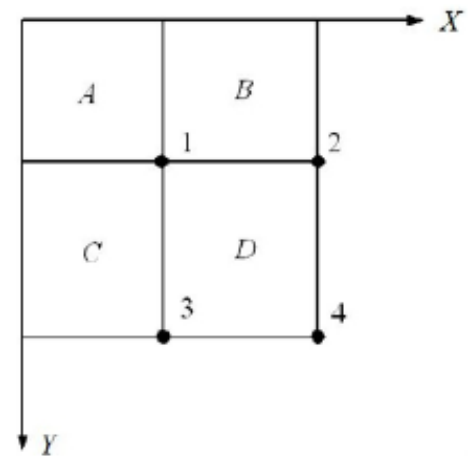

Fig. 1 Specific Point Integral Image

According to the figure above, the eigenvalue can be calculated in the order of Point 2, Point 3 and Point 4:

$$
\begin{gathered}
i i_{2}=\operatorname{sum}(\mathrm{A})+\operatorname{sum}(\mathrm{B}) \\
i i_{3}=\operatorname{sum}(\mathrm{A})+\operatorname{sum}(\mathrm{C}) \\
i i_{4}=\operatorname{sum}(\mathrm{A})+\operatorname{sum}(\mathrm{B})+\operatorname{sum}(\mathrm{C})+\operatorname{sum}(\mathrm{D})
\end{gathered}
$$

According to the formula, the value of each endpoint is calculated from the integral figure, representing a rectangle, and then the value of each endpoint is calculated in addition and subtraction. The size and position of the rectangle to be calculated are not related to the computation time, even though this computation time is a constant, and this is the greatest advantage of integral image calculation.

Adaboost algorithm and cascade classifier. Adaboost algorithm is a classification algorithm, which is proposed by Freund and E.Schapire Robert in 1995. The main idea is to use weaker classifier to boost, which constitutes a strong classifier with strong classification ability.

(1)Generates weak classifier out of expanding Haar features. The infrared backlight camera was taken into the gray scale, and the weak classifier was trained by the gray level image. A weak classifier can make a classification of any single feature or combination of features, and its main function is to make a two value judgment on the candidate region. Specific definitions are as follows:

Where $\mathrm{X}$ window detection, $\mathrm{f}(\mathrm{x})$ for calculating the eigenvalue of the rectangular function, $\theta$ is through the threshold of the training of weak classifiers, $\mathrm{P}$ denotes inequality direction, the value of +1 . The map of each feature $\mathrm{F}$, training corresponds to a weak classifier $\mathrm{H}(\mathrm{x}, \mathrm{F}, \mathrm{P}, \theta)$ and determine the optimal threshold, making the weak classifier $\mathrm{H}(\mathrm{x}, \mathrm{F}, \mathrm{P}, \theta)$ of all the training samples classification error rate is the lowest.

The training process of a weak classifier are as follows:

1) calculated for each feature of $f_{i} \quad(i=1 \ldots m)$ ( $\mathrm{m}$ is the total features) corresponding to the characteristic values of the training sample

2) sort all the eigenvalues; 
3) each element of a sequence of good order:

$\mathrm{T}+$ and weight of all positive cases are calculated;

$\mathrm{T}$ - and weight of all negative cases are calculated;

The calculation of Sij+ and weight of positive examples of the element before

The calculation of Sij- and weight of negative examples of the element before

4) The classification error of the feature value Fk is as follows: the value of a feature value Fk in front of it is a threshold, the classification error of the classifier is:

$\varepsilon_{i}^{j}=\min \left(S_{i}^{j^{+}}+\left(T^{-}-S_{i}^{j^{-}}\right), \quad S_{i}^{j^{-}}+\left(T^{+}-S_{i}^{j^{+}}\right)\right)$

5) Calculation of minimum weak classifier error $\varepsilon j$, also recorded at this time if the classifier direction symbols $\mathrm{P}$ and threshold $\theta$ and classifier of rectangle features.

$\varepsilon_{i}=\min \left\{\varepsilon_{i}^{j}\right\}=\min \left\{\min \left(S_{i}^{j^{+}}+\left(T^{-}-S_{i}^{j^{-}}\right), \quad S_{i}^{j^{-}}+\left(T^{+}-S_{i}^{j^{+}}\right)\right)\right\}$

Special note: in the pre prepared training sample, the sample size is required to be normalized and gray to $20 * 20$, so that the sample size of each gray image is consistent, which ensures that every Haar feature is in a sample.

(2)Strong classifier training. In the process of training strong classifier, $\mathrm{T}$ is represented by a number of weak classifiers which are contained in the strong classifier. Of course, if it is a cascade classifier, the number of weak classifiers in the strong classifier may be relatively small, and the number of strong classifiers can be concatenated. The following steps are as follows:

Given a series of training samples $\left(x_{1}, y_{1}\right) .\left(x_{2}, y_{2}\right) \ldots . .\left(x_{n}, y_{n}\right)$, where $y_{i}=0$ denotes negative sample (non face), $y_{i}=1$ is represented by positive sample (face), $\mathrm{n}$ is a total number of training samples.

1)initialization weights $w_{t, i}=D(i)$;

2)normalized weight of $t=1 \ldots . . . T$,

$$
q_{t, i}=\frac{w_{t, i}}{\sum_{j=1}^{n} w_{t, j}}
$$

3) For each feature $\mathrm{F}$, training a weak classifier $h(x, f, p, \theta)$ : Calculation of feature correspondence if separator weighted $\left(q_{t}\right)$ error rate $\varepsilon_{i}$.

4) The minimum error $\varepsilon j$ is calculated, and the $P$ and the threshold $\theta$ of the weak classifier are recorded.

$$
\varepsilon_{i}=\min \left\{\varepsilon_{i}^{j}\right\}=\min \left\{\min \left(S_{i}^{j^{+}}+\left(T^{-}-S_{i}^{j^{-}}\right), \quad S_{i}^{j^{-}}+\left(T^{+}-S_{i}^{j^{+}}\right)\right)\right\}
$$

After $\mathrm{T}$ iterations, the $\mathrm{T}$ optimal weak classifier $\mathrm{H} 1 \mathrm{x}$ ) hT $(\mathrm{x}, . .$.$) in the following way can be$ combined into a strong classifier.

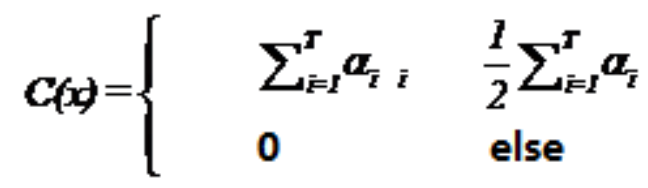

$$
\begin{aligned}
& \text { where } \alpha_{\mathrm{i}}=\log \frac{1}{\beta_{i}}=\log \frac{1-\varepsilon_{i}}{\varepsilon_{i}}=-\log \varepsilon_{i}
\end{aligned}
$$

Then, the strong classifier to an image to be detected, the equivalent of all the weak classifiers vote, then the results of the vote in accordance with the weak classifier error rate weighted sum, by comparing the weighted sum of the voting results and the average vote count. If it is greater, calculate in the strong classifier, and if it is less, exclude to get the final the results.

$$
\frac{1}{2}\left(\sum_{i=1}^{T} \alpha_{i} \cdot 1+\sum_{i=1}^{T} \alpha_{i} \cdot 0\right)=\frac{1}{2} \sum_{i=1}^{T} \alpha_{i} \cdot 1
$$

(3) Construct the cascade AdaBoost algorithm. The core of the cascaded AdaBoost is in the process of the algorithm for the $\mathrm{T}$, and the weak classifier is selected from each of the weak classifiers in the iterative training process, and the $\mathrm{T}$ is connected to a strong classifier according to certain 
criteria. If the correct rate of the weak classifier is only a little bit, the correct rate of the classifier can be improved greatly. The output value of the continuous AdaBoost algorithm is spread over the whole real number field. Therefore, the probability distribution of all the classifiers can be simulated.

The cascaded classifier is a strong classifier which is trained by the continuous AdaBoost algorithm. Finally, the weak classifier will be selected automatically according to their classification performance.

Generally speaking, in the detection of the video image, the target can be detected only a very small part of the image to be measured, but in the image, there are a lot of interference to hinder the detection of targets. We quickly remove the interference or non target areas and leave most of the time to detection to remove the interference of the target window. This will not only improve the target detection speed, and improve the accuracy of target detection. The construction of cascaded AdaBoost algorithm is the algorithm we need.

\section{Dimming Principle.}

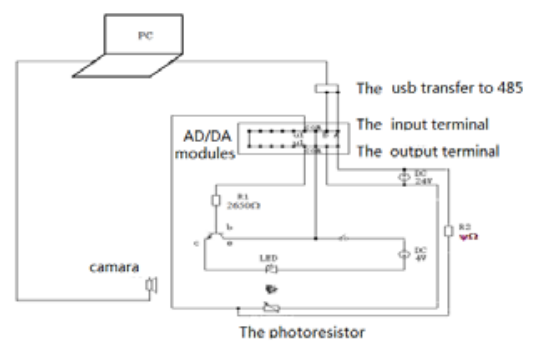

Fig. 2 Divice Connection Diagram

The use of high-definition infrared backlight camera, a person's face, with the help of computer vision library provided by OPEN-CV Intel, AdaBoost algorithm and cascade classifier, which has high accuracy and fast features to find the face, and finally found the pupil. The size of the iris is not changed, the relative size of the iris and the pupil is not changed with the distance between the eyes and the light, so the relative size of the pupil can be calculated with the iris.According to the pupil size and the corresponding relationship between the light and the need to output a suitable value to the voltage source module, and then change the voltage across the resistor, change the current through the resistor, through the amplification of the current amplifier, the LED table lamp. In addition, in order to reflect the absolute intensity of the desktop, LED lighting, there is a photosensitive resistance, light resistance sensing at this time, will get a corresponding resistance value, and the voltage meter will be parallel with the photosensitive resistor to the computer, the computer determines whether to meet the requirements of eye comfort, if not reached, then fine tune intensity, until the light is most suitable for the human eye.

\section{Test and performance analysis}

Value illuminance relationship. According to the experimental circuit, the circuit is connected with the lamp to the top of the desktop, and the lamp to the top of the desktop, open the computer, open the light meter, run the corresponding program, from the 600-4000 every 100 input values, record the corresponding data, can be drawn from the LED lamp Y / Lux and the input data to the number of analog converter $\mathrm{x}$ is a positive correlation, the computer fitting function is $\mathrm{y}=361.1 \ln (\mathrm{x})$ -2217.8. LED light emitted from light intensity value Y / Lux and through the LED lamp current value $\mathrm{x} / \mathrm{Ma}$ is related to the relationship, the computer fitting correspondence function expressions for the $y=2.7975 x+48.092$.

Pupil diameter and illuminance. Pupil's role is to change the amount of light, and luminous flux is a function of illumination, and illumination and pupil diameter between established contact, pupil diameter $R$ is function of the eye light illumination 10: $r=f(L 0)$. To keep the light and the eye distance is $50 \mathrm{~cm}$, the light is the only light source, the light and the pupil detection instrument, continuously adjust the light, so that the pupil in the light from the 100lux -780 lux per 40lux unit increments, record the corresponding pupil diameter in the form, get the corresponding curve. 


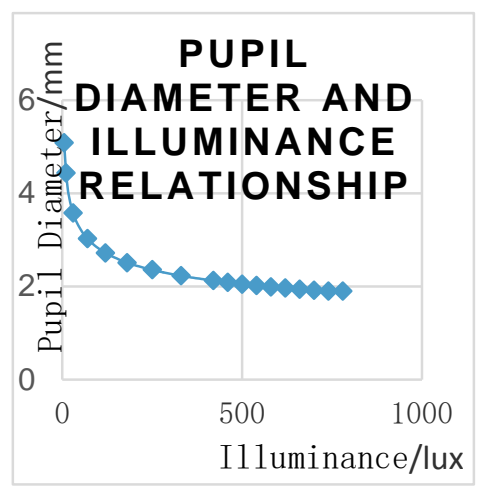

Fig 3. Pupil Diameter and Illuminance Relationship

From the relationship between pupil diameter and illumination, we can see that the diameter of the pupil is $\mathrm{Y} / \mathrm{x} / \mathrm{lux}$ and the intensity of the light emitted from the LED lamp is negative correlation, and the corresponding relation function expression is $y=6.99 x-0.197$

The distance between the light and the photosensitive resistor is $50 \mathrm{~cm}$ constant, the light is the only light source, the light source is opened, the program is running, and the light is continuously adjusted, so that the illumination of the light source is increased from 5lux-1000lux per 40lux. Relationship as shown in figure.

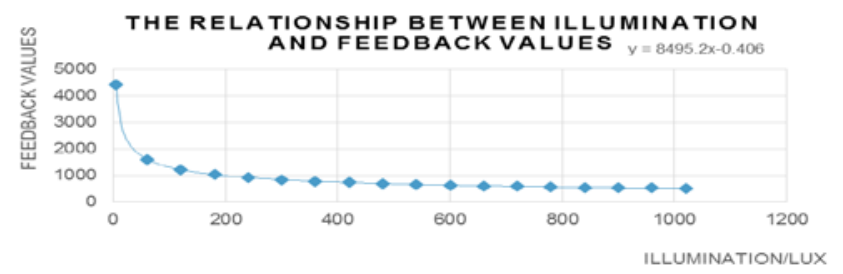

Fig. 4 The Relationship Between Illumination and Feedback Values

From the illumination and the numerical diagram, we can see that the value of Y and LED light intensity lux / $\mathrm{X}$ negative correlation, the computer fitting the corresponding relationship function expression is $y=8495.2 x-0.406$.

\section{Summary and Outlook}

The first step to the application of pattern recognition in the study of adaptive lamps. Human nature of the design so that it can according to the state of the eye to achieve the light intensity of the automatic adjustment, the right of the human eye to achieve the most comfortable state, better protection of the eyes, to prevent eye diseases. The same principle and algorithm can be applied to the electronic products of the screen brightness control. Currently on the market, the screen brightness adjustment is mainly through the light sensitive element and photosensitive sensor induction, but the idea proposed in this paper can achieve the screen brightness for the eye of the adaptive adjustment, is the existing products can not match.

\section{References}

[1] H.B. Du, Research and implementation of algorithm for iris location and recognition, Shanghai Jiao Tong University, 2013

[2] Q.C. Tian, L. Xu, Z.H. Lin, Research on iris encoding algorithm based on local edge detection , Computer application Research, 2005, 08:230-232+257

[3] W.Q. Fan, L. Xu, Z.H. Lin, Iris localization algorithm based on gray distribution of human eyes, Photoelectron and laser, 2006, 02: 226-230

[4] Z. Zhang, Y.P. Wang, Digital image processing and vision, People Post and Telecommunications Press, 2010

[5] H.L. Zhang, Visual C++ digital image pattern recognition technology and engineering practice, People Post and Telecommunications Press, 2003 